\title{
Targetoid Lesion
}

National Cancer Institute

\section{Source}

National Cancer Institute. Targetoid Lesion. NCI Thesaurus. Code C111972.

A red, edematous ring skin finding that may have a central dusky disk and an erythematous halo. 